\section{An oregonian school of zebra fish}

A group of neuroscientists at the Institute of Neuroscience, University of Oregon, use what is rapidly becoming a new standard embryological preparation, the zebra fish (Fig. 1), an animal that develops rapidly and is small and optically clear. Five recent papers from this group are summarized below; they deal with the onset of developmental restrictions, pathfinding by motor axons, and the development of the lateral line placode.

The late George Streisinger of the University of Oregon first promoted the zebra fish as particularly suitable for developmental and genetic studies. Some of his expectations are now being realised by others at the same institution.

Charles Kimmel and his associates have employed the cell marking techniques originally developed for use on the leech by David Weisblat, Gunter Stent, and their collaborators. Briefly, individual cells of the embryo are injected with fluorescent molecules (fluorescein or rhodamine conjugated to dextrans or horseradish peroxidase) that are subsequently passed on to the daughter cells. Thus, fluorescence unambiguously implies a cell's descent from an injected one. The embryo is then observed in real time as it develops. The fluorescent cells are visualized on a television screen coupled through a SIT camera to a fluorescence microscope. This equipment allows very low levels of incident radiation to be used since levels high enough to visualize the cell directly would kill it. Earlier work had shown that when blastomeres were injected, their progeny dispersed throughout the embryo and ultimately settled in a wide variety of tissues (nerve, muscle, epidermis, etc.), where they were intermixed with unlabelled cells from different clones.

In a recent paper, Kimmel and Warga ${ }^{1}$ injected blastomeres, as before, but then waited until gastrulation before observing the cells in detail. They focused on one or several wellisolated fluorescent cells, and then monitored their fates. In most cases, all members of a subclone (the descendants of one of these isolated groups) settled in the same tissue - the two exceptions were probably neural crest cells. Fig. 2 summarizes the results of one such set of observations, typical of 13 others. The founder cell was injected in a 512-cell blastula at about 3 hours after fertilization. By 7 hours (midgastrula stage) two of the descendants, probably siblings, had settled in the neural tube where both divided and then dispersed longitudinally to end up as four clusters of labelled cells on both

For technical reasons we are unable to reproduce this figure in colour. See the June issue of Trends in NeuroSciences for full colour illustration. sides of the midline.

These results show that the fate of a clone, i.e. the tissue that it will form, is restricted around the time of gastrulation, and that the maintenance of this restriction does not depend on the members of the clone remaining together. It is difficult to imagine how such clear cut conclusions could have been drawn with any other experimental technique. Moreover, it is likely that the zebra fish is particularly suited to this technique, since members of blastomeric clones are dispersed so widely during epiboly (the overgrowth of the surface of the embryo by presumptive ectoderm), that visually isolated single cells probably appear more frequently than, for example, in amphibian larvae, which develop without epiboly.

The development of spinal motor neurons has been described in three additional papers ${ }^{2-4}$. In the first two, Eisen, Myers and Westerfield observed the growth cones of primary motor neurons. (The ventral horns of amphibians and fish have long been described as having two types of motor neurons, primary and secondary, the former being large, few in number and early to develop, relative to the latter.) In zebra fish, each segment has only three primary motor neurons, arranged rostrocaudally. They were observed in vivo, either with Nomarski optics on unstained material, or with fluor- escence optics in embryos that had been injected as blastulas with one of the fluorescent markers. Axonaloutgrowth was strikingly stereotyped. The growth cone of the caudal primary motor neuron was always the first to leave any segment of the spinal cord, and it always followed the same path to terminate in the ventral part of the hypaxial musculature*. Then the middle primary motor neuron's growth cone emerged, tracked its predecessor for a short distance, and then charted its own pathway into the epaxial musculature. Last to emerge was the growth cone of the rostral primary motor neuron; it followed its two predecessors initially and then diverged to ramify in the dorsal portion of the hypaxial musculature. All primaries of a given type followed the same trajectory in any segment. All three termination fields were restricted to the segment of origin.

These papers provide the first description of the behavior of single motor axons in the vertebrate nervous system, in contrast to the best previous work, in chick, which dealt with populations of axons ${ }^{5,6}$. Moreover, the work in fish is done in real time, and in an intact embryo.

These events are very reminiscent of axonal pathfinding in the grasshopper $\mathrm{CNS}^{7}$. In both cases, the particular neurons are identified uniquely (a hitherto uncommon occurrence in vertebrates), and their trajectories and terminations are very predictable - even certain subtleties of their behavior resemble the grasshopper's $\mathrm{G}$ and $\mathrm{C}$ neurons. For instance, the middle primary's growth cone typically pauses

* The axial musculature is divided dorsoventrally by a horizontal septum into hypaxial and epaxial on the ventral and dorsal sides, respectively.

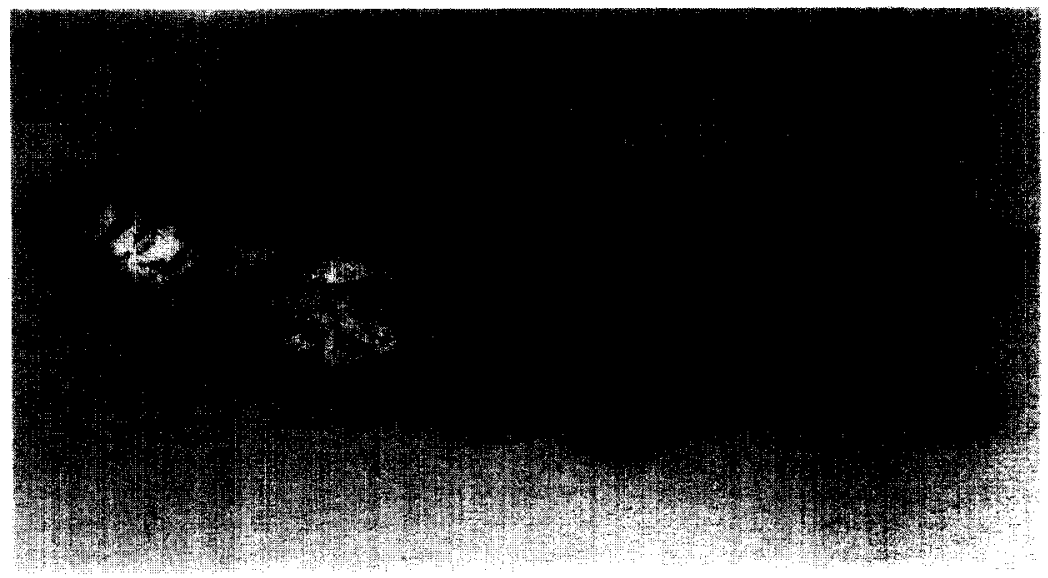

Fig. 1. A zebra fish (C) 1986. Elsevier Science Publishers B.V.. Amsterdam 0378-5912/86/\$(12.01 


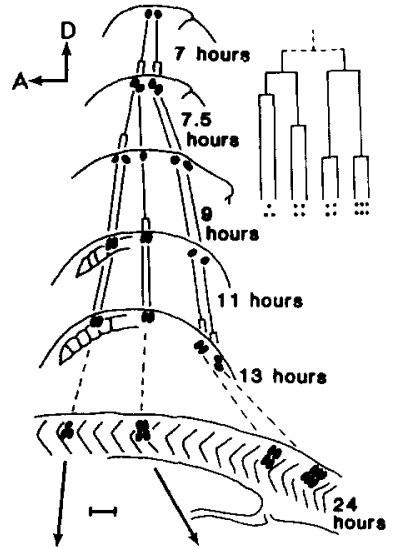

Fig. 2. Schematic drawing of a neural cell lineage. The embryo is sketched as if seen from the left side ( $A$, anterior; $D$, dorsal). The founder cells at 7 hours divided and dispersed longitudinally through the neural tube until the observations stopped at 24 hours, at which time there were four clusters of cells, each with members on both sides of the midline. Scale bar is $100 \mu \mathrm{m}$. (Taken, with permission, from Ref. 1.)

for about half an hour at the horizontal septum, then sends branches both dorsally and ventrally, and finally maintains the dorsal branch and retracts the ventral one. These observations support the idea that vertebrates and insects, and probably other invertebrates as well, share similar mechanisms of pathfinding. It is also worth noting that the idea of identified neurons, so effectively used in studies of invertebrates, had not been thought to be applicable to vertebrate nervous systems except for the relatively rare cells such as Mauthner and Muller cells in the medullas of anamniotes. But when the zebra fish spinal cord is examined early, at a time when only the primaries have appeared, and at the segmental level, the neurons are clearly identifiable as individuals. Later addition of secondary motor neurons ${ }^{4}$ makes the tissue more complex, but the uniqueness of these first three neurons is not affected.

The exclusive domains laid down by the primary growth cones are maintained into adulthood. Westerfield, McMurray and Eisen ${ }^{3}$ recorded from muscle cells and motor neurons in larval and adult fish, and marked the motor neurons as well. The three segmental primary motor neurons were still evident, and their fields of innervation were as exclusive and restricted as their embryonic fields. Fig. 3 illustrates these domains, and includes the field of a secondary motor neuron as well. Unlike the primary, it crosses segmental boundaries, though usually not more than one. This qualitative difference between the two kinds of motor neurons also extends to their innervation of muscle cells; each muscle cell was innervated by only one primary motor neuron, and up to three secondaries.

In related work, Metcalfe ${ }^{8}$ studied axonogenesis in the lateral line organ. In amphibians, the lateral line placode (on the embryo's head) gives rise to migratory primordia, groups of cells that migrate away from the placode and leave a few cells behind at various points along this migration. These cells become the specialized receptors that form the neuromasts of the lateral line. Other cells in the placode become intracranial ganglion cells with their

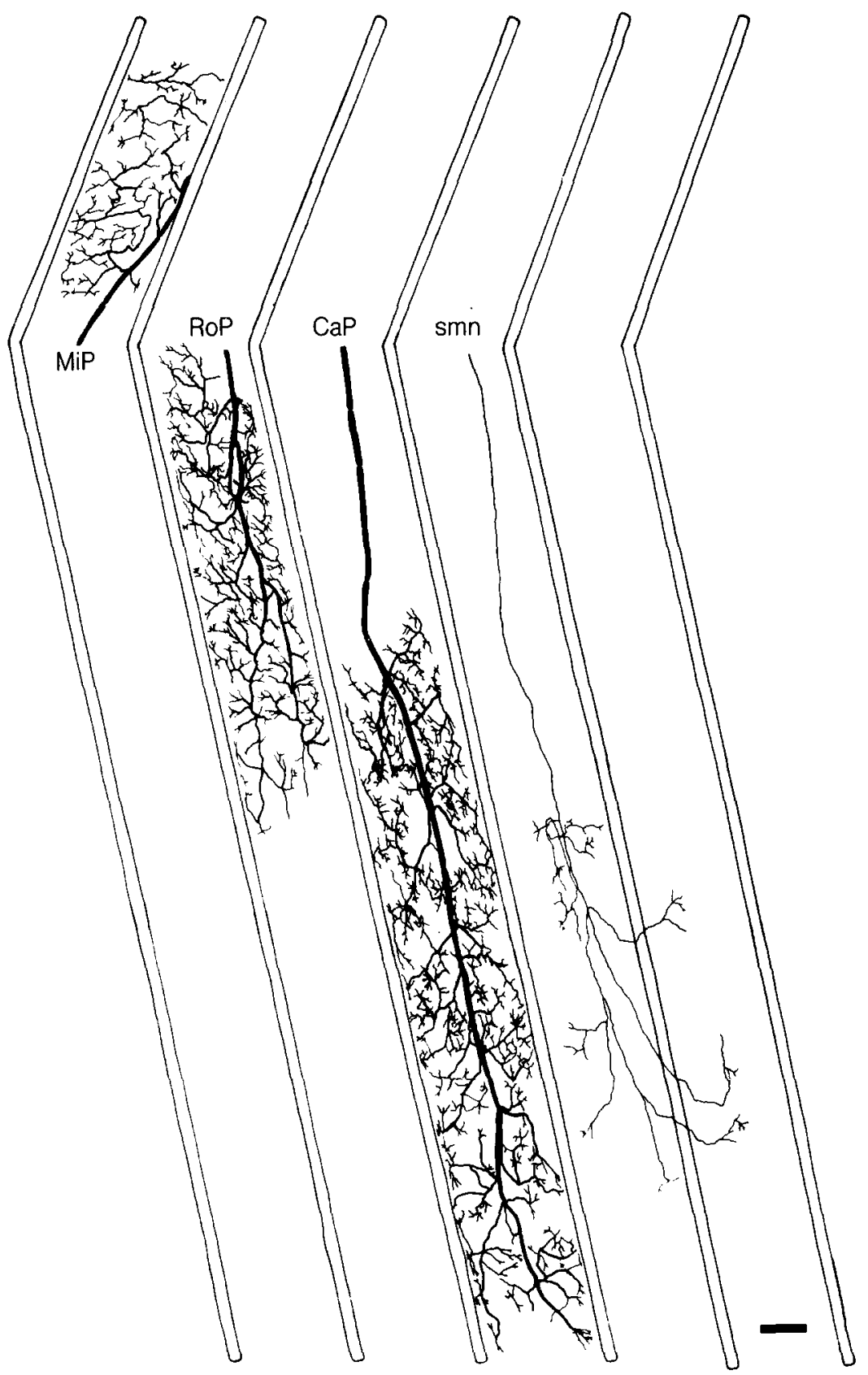

Fig. 3. A sketch of the terminal fields of fourtypes of motor neurons in the adult. The vertical bars represent the ribs. Dorsal is up, anterior left. Each segment had all four types of motor neurons innervating it, but only one is shown per segment. MiP, middle primary motor neuron; RoP, rostral primary motor neuron; $C a P$, caudal primary motor neuron; smn, secondary motor neuron. Scale bar is $300 \mu m$. (Taken, with permission, from Ref. 4.) 
peripheral process extending along the lateral line, innervating neuromasts. Metcalfe has studied the development of the posterior lateral line in zebra fish, confirmed that it resembles the amphibian case, and has uncovered an elegantly simple plan of development. He used scanning EM and light microscopy with HRP for tracing axons, and confined his observations to the period 18-48 hours post-fertilization. The migratory primordium was visible on the animal's flank as a bump that progressed from the head to the tip of the tail, a distance of about $2 \mathrm{~mm}$, in 22 hours. The growth cones of the presumptive ganglion cells chased the migrating cells; indeed, one of the growth cones was often in among the migrating cells, with others strung along behind. Thus, from the beginning, the neuritic link from ganglion cell to presumptive neuromast is present. The neurites do not have to find their target; instead, they travel with it and lay down the cable that later neurites will follow. It is possible that this may prove to be the general pattern for the development of placodally derived structures innervated by ganglion cells.

These three diverse topics: restriction of cell fates, axonal pathfinding by motor neurons, and development of the lateral line, have all been impressively clarified by these careful studies on the zebra fish. It seems likely that the favorable preparation and the elegant techniques will continue to increase our understanding of old problems and our awareness of new ones.

\section{Selected references}

1 Kimmel, C. B. and Warga, R. M. (1986) Science 231, 365-368

2 Eisen, J. S., Myers, P. Z. and Westerfield, M. (1986) Nature 320, 269-271

3 Myers, P. Z., Eisen, J. S. and Westerfield, M. J. Neurosci. (in press)

4 Westerfield, M., McMurray, J. V. and Eisen, J. S. J. Neurosci. (in press)

5 Lance-Jones, C. and Landmesser, L. (1981) Proc. R. Soc. London Ser. B. 214, 1-18

6 Hollyday, M. (1980) Curr. Top. Dev. Biol. 15 , 181-215

7 Raper, J., Bastiani, M. J. and Goodman, C. S. (1983) J. Neurosci. 3, 31-41

8 Metcalfe, W. (1985) J. Comp. Neurol. 238 , $218-224$

STEPHEN S. EASTER, JR.

Division of Biological Sciences, University of Michigan, Ann Arbor, MI 48109, USA.

\section{Structure and function in synapses: emerging correlations}

Recent observations on membrane structure at neuromuscular junctions of vertebrates and arthropods suggest that a class of prominent membrane particles at the active zone, tentatively identified as calcium channels, can be reliably discerned. These particles are packed more densely at synapses which are thought to release more transmitter, and in some instances occur in greater numbers at such synapses. $A$ 'structure-function' hypothesis derived from these observations is that the probability of release of a quantal unit of transmitter (synaptic vesicle?) is enhanced by having more membrane calcium channels close to the site at which transmitter is released. Variation in performance among different synapses could be determined in part by the pattern of distribution of presynaptic calcium channels.

Behavioural acts are regulated by synapses, both central and peripheral. The characteristics of many responses can be attributed to properties of synapses involved in the neural networks governing the response. Thus, a research goal of continuing interest is to elucidate the factors that determine synaptic performance.

One approach to the problem is to examine synaptic ultrastructure and to see whether function can be correlated with it. To be successful and convincing, this approach demands that ultrastructural information be obtained from the same synapses used to provide measurements of physiological efficacy. Few investigations have met this test at a truly rigorous level. However, recent studies of both vertebrate ${ }^{1}$ and invertebrate $^{2}$ synapses have provided new information on membrane structure which could lead to a more complete understanding of how synaptic function depends on structure.

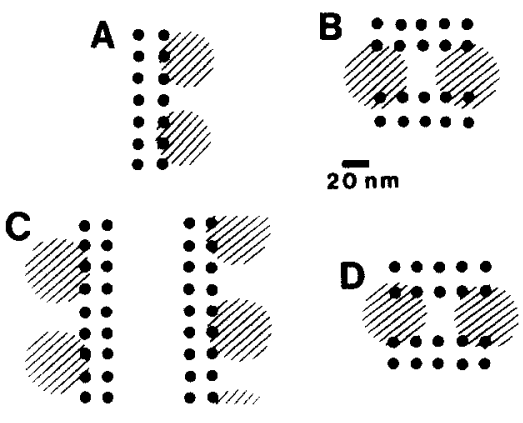

Fig. 1. Arrangement of prominent membrane particles (putative calcium channels) at active zones oflizard tonic (A) and phasic (B), frog phasic (C), and rat (D) neuromuscular junctions (after Walrond and Reese ${ }^{J}$ ). Active zone particles (small solid circles) and associated synaptic vesicles (large hatched circles) are drawn to scale. The double array of particles at lizard phasic (B) and rat phasic (D) neuromuscular junctions could provide more intense calcium entry at the site of a synaptic vesicle, and consequently a greater probability of release of the vesicle. Scale bar is $20 \mathrm{~nm}$.
Peripheral synapses (especially neuromuscular junctions) are easiest to study in this correlative fashion. They provide a wide range of synaptic performance, are accessible for detailed ultrastructural investigation, and are important in locomotion and behaviour, being the final necessary synaptic component of the motor system. Neuromuscular junctions of arthropods are very diverse in performance, and offer good models for the less accessible central synapses. Vertebrate neuromuscular junctions, though generally specialized for high output of transmitter, also show considerable diversity in transmitter-releasing properties, and provide good material for freezefracture investigations of membrane structure.

In their studies of neuromuscular junctions of lizard (Anolis) intercostal muscles, Walrond and Reese ${ }^{1}$ compared junctions on tonic muscle fibers with those on twitch (phasic) muscle fibers. Neuromuscular transmission in twitch fibers involves release of enough transmitter to drive the membrane potential past the threshold for generation of a propagating muscle action potential. In tonic fibers of amphibians and reptiles summating junction potentials, rather than action potentials, control contraction, and the junctional output of transmitter for a single presynaptic action potential is about 10 times less ${ }^{3}$. There is evidence for structural features which correlate with the difference in transmitter output.

At vertebrate neuromuscular junctions, ultrastructural studies have given rise to the concept of the active zone, characterized by a double row of prominent membrane particles on the transmitter-releasing surface of the nerve terminal, together with associated intracellular synaptic vesicles and presynaptic 'dense bodies' or 'dense projections'. If one accepts the hypothesis, supported by a large body of 